\title{
Shape design optimization of road acoustic barriers featuring top-edge devices by using Genetic Algorithms and Boundary Elements*
}

\author{
R. Toledo, J. J. Aznárez, D. Greiner, O. Maeso \\ Instituto Universitario de Sistemas Inteligentes y Aplicaciones Numéricas en Ingeniería \\ (SIANI) Universidad de Las Palmas de Gran Canaria \\ Edificio Central del Parque Científico y Tecnológico \\ Campus Universitario de Tafira, 35017, Las Palmas de Gran Canaria, Spain \\ \{rtoledo, jjaznarez, dgreiner, omaeso\}@siani.es, web: http://www.siani.es
}

June 2015

\begin{abstract}
This paper presents a Boundary Elements (BE) approach for the efficiency improvement of road acoustic barriers, more specifically, for the shape design optimization of top-edge devices in the search for the best designs in terms of screening performance, usually represented by the insertion loss (IL). With this aim, a procedure coupling BE with Evolutionary Algorithm is proposed in pursuing barrier configurations with ever higher IL. The complexity normally associated with such designs raises the need to consider some geometric simplifications in order to ease the shape optimization processes. In this way, the overall barrier configuration is modeled as both thickness and null-thickness bodies (the boundary thickness is neglected), as representatives of very thin elements. Such an idealization requires a Dual Boundary Element formulation that allows the problem to be solved. The procedure is applied to $2 \mathrm{D}$ problems and numerical results are presented on the basis of simulations on noise barriers with three different top designs. It is a quite simple process that makes use of well-known both formulations and procedures. The improvements observed in the designs obtained invite to further studies in the same line on devices with similar applications.
\end{abstract}

Keywords: Noise barriers, Very thin bodies, Shape optimization, Genetic Algorithms, Dual Boundary Element Formulation.

\section{Introduction}

The inclusion of sound barriers for abating the negative effects of road traffic noise near residential areas is a broadly used strategy. Considerable research works and studies focused on sound diffraction around barriers have been conducted in the past two decades, specifically in the prediction of the

${ }^{*}$ This is the pre-peer reviewed version of the following article: Toledo R, Aznarez JJ, Greiner D, Maeso O. Shape design optimization of road acoustic barriers featuring top-edge devices by using Genetic Algorithms and Boundary Elements. Eng Anal Bound Elem 2016;63:49-60, which has been published in final form at http://dx.doi.org/10.1016/j.enganabound.2015.10.011 
performance and the development of more efficient designs. Among all of the different theoretical methods proposed concerning the issue, the Boundary Element Method (BEM) has been broadly used for its indubitable benefits in the analysis of exterior sound propagation problems [1 $[6]$. More specifically, a comprehensive, up-to-date revision of the application of this technique in the assessment of the screening behavior on various barrier designs in different scenarios can be consulted in [7]. Some of these research works focus on both the application and the potential of the standard BEM formulation for the aforementioned cases. Others deal with the incorporation of some geometric changes on classic, widely used barrier configurations (such as $\mathbf{Y}-$, T-, $\mathbf{M}$ - and arrow-shaped barriers, multiple-edge screens, etc.) and the assessment of their influence on the acoustic performance. In this line, with the purpose of searching designs more and more acoustically efficient, the shape optimization methods (extensively used in other engineering fields [8]), are presented as valuable application tools. Such is the case of Evolutionary Algorithms (EA) and, in particular, Genetic Algorithms (GA), which coupled with BEM have been successfully implemented in the design optimization of noise barriers in exterior acoustic problems within the SIANI Institute, where this work is developed [9 13]. Some other notably contributions concerning the issue can be found in the bibliography. For instance, with a markedly academic nature, Duhamel [14] performs the optimization of a noise barrier starting off with a prismatic, volumetric structure built of equally sized bricks to lead to the final optimized shapes with non-inner holes and fillings. As a result, some bricks remain from the original prismatic configuration, while others are removed according to the patterns established by the process. Other interesting works present a more practical approach. In this line, Baulac et al. [15] propose an original optimization method for bi-dimensional multiple-edge noise barriers based on the adjusting of both some geometric parameters and the impedance values of some boundaries. The same authors study the performance of $\mathbf{T}$-shaped barriers featuring a reactive surface on the top [16]. Still on the same practical approach, Grubeša et al. [17] broaden these methodologies into a three-dimensional analysis involving a multi-objective optimization of both the acoustic performance and the economic feasibility of noise barriers made of varying cross-section modules. Concerning the authors of this work, a monoand a multi-objective optimization process for bi-dimensional problems, following a procedure in the line of the one introduced here, can be consulted in Greiner et al. [11]. More recently, the scope of such work has been broaden by considering the shape optimization of very thin barriers using a Dual BEM formulation (Toledo et al. [13]).

In relation to the previous work done, this paper deals with an improved, more advanced procedure that allows the shape design optimization of more complex road barrier designs to be easily accomplished. In this line, a more general BE formulation has been developed and implemented in a computer code that allows a procedure with a broader scope. This enables us to tackle the shape design optimization of complex edge-modified barriers in a way, to the authors' knowledge, not covered so far in the bibliography concerning the issue. As an application, numerical results on the basis of three models with complex top designs featuring both thick and very thin bodies (idealized as null-thickness type) are performed. In this respect, the Dual formulation arises as the unique valid strategy of BEM that allows us to assume such a simplification of reality, greatly facilitating the geometric definition of complex configurations with no substantial influence on the acoustic performance for the considered thickness of very thin bodies [18].

Two-dimensional sound propagation hypotheses are considered, i.e., an infinite, coherent monofrequency source of sound and a noise barrier with no geometric variation that stands on a flat plane 
(ground) of uniform admittance. The problem is performed in the frequency domain with the usual assumptions (Helmholtz equation): the medium (air) is modeled as homogeneous, elastic and isotropic with no viscosity, under small disturbances and initially at rest with no wind effects. Expression of the objective function to be maximized throughout the shape optimization process is written in terms of this response.

The work is structured as follows: after this introduction, in section 2 a detailed description of the top barrier designs to be studied is provided, including a brief state of the art, the scattering and the screening properties as well as the use and implementation of well- and diffuser-based top designs in exterior acoustic problems. In section 3, the modeling and discretization by implementation of a Dual BEM formulation is described. Section 4 deals with the shape optimization procedure. Finally, section 5 shows results and discussion, and section 6 covers the conclusions of the paper.

\section{Diffuser-based top designs in exterior acoustic problems}

For their unquestioned benefits for scattering sound field, the use of diffusers has been the subject of many reviews and studies in indoor acoustic projects. Among them, those based on sequence number series (such as Maximum Length Sequence (MLS), Quadratic Residue Diffuser (QRD), Primitive Roots Diffuser (PMR), etc.) have gained prominence for their excellent scattering properties, characterized by an approximately flat power spectral density. As the power spectrum and surface scattering are closely related [19 21], the far field scattering can be approximately predicted by taking the Fourier transform of the surface reflection coefficients (in this case, a series of wells that could be modeled as a flat surface of varying impedance). In short, any numerical sequence featuring good auto-correlation properties (in other words, the auto-correlation function of the reflection coefficients of the surface is a delta function) presents a Fourier transform with a flat power spectral density, meaning that such a surface exhibits an even scattering distribution of sound.

With the purpose of raising the acoustic performance, numerous and innovative designs have been proposed and studied in the literature for compensating the limitations normally associated with the parameter with greatest influence on the barrier efficiency: the effective height. In this way, despite the indoor-oriented application of well-based designs, the use of such devices on noise barriers in exterior acoustic problems has evidenced a good performance when compared with both a vertical screen and other top configurations. Some noteworthy works concerning the use of diffusers installed on the barrier top can be found in the literature. Such is the case of those based on mathematical number sequence, such as Quadratic Residue Diffusers (QRDs) [22 25] and Primitive Roots Difussers (PRDs) [26]. Other designs featuring elaborated configurations eligible for either some kind of scattering or screening behavior can be found in [27, 28] (see Figure 1).

All the aforementioned works address the problem with the standard BEM formulation, considering the real geometry of the barrier comprised of thick and very thin elements. Despite their remarkable contribution, no shape design optimizations are performed in the referenced works. In this regard, the methodology here presented proposes a general procedure that aims at optimizing the shape design of edge-modified road acoustic barriers using BEM. This methodology offers an appropriate, ideal solution for complex configurations eligible for some sort of geometric simplification. Under this proposal, the overall barrier configuration can be modeled considering both thickness and null-thickness bodies as representatives of very thin elements (the thickness of these elements is neglected). Based on a frame 
of free geometric constraints, the definition of the barrier profile is then easily accomplished. This approach results in faster computational times within a cumbersome process where every possible design is assessed along the whole spectrum of frequencies.

(a)

REAL VOLUMETRIC GEOMETRY

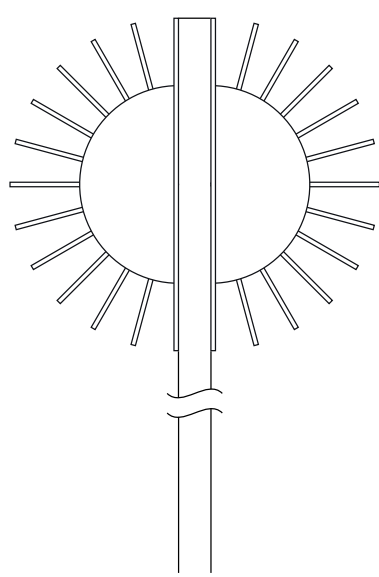

(b)

VOLUMETRIC GEOMETRY WITH IDEALIZED ELEMENTS

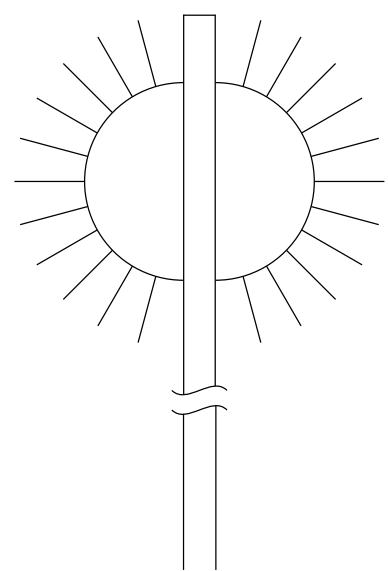

REAL VOLUMETRIC GEOMETRY

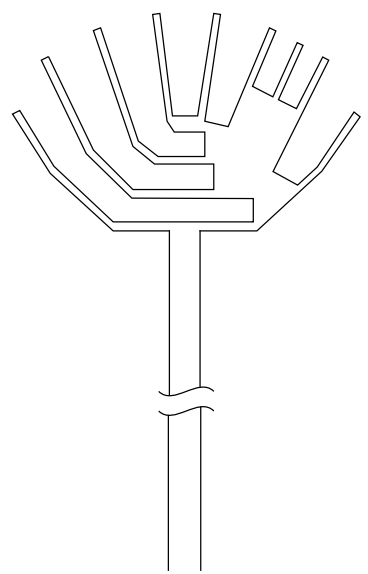

VOLUMETRIC GEOMETRY WITH IDEALIZED ELEMENTS

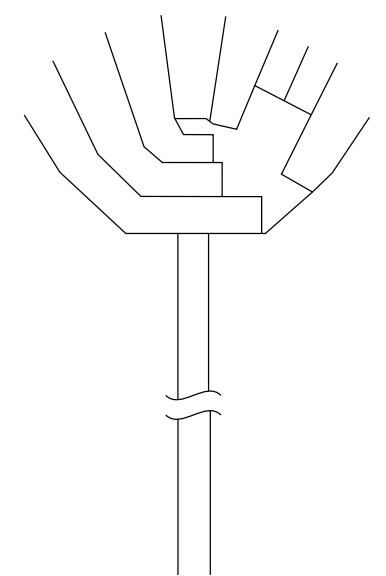

Figure 1: Examples of complex designs eligible for undergoing geometric idealizations. (a) Waterwheeltop barrier from Okubo and Fujiwara [27]. (b) Complex barrier-top featuring wells with different lengths and paths [28].

The need of the implementation of the Dual BEM formulation in this work is clarified in Figure 2, The strategy of the application of both formulations varies depending of the nature of the element under consideration. This way, with the purpose of mitigating the effects of the fictitious eigenfrequencies when dealing with non-thin bodies, a Dual BEM formulation based on the combined use of the standard boundary integral equation (SBIE) and the hyper-singular boundary integral equation (HBIE) coupled by means of a frequency-related complex value is proposed [29]. The nature of the issue is different when dealing with very thin bodies. In this case, numerical integration problems may appear affecting, equally, to the barrier performance. The idealization of such elements as non-thickness bodies not only solves the problem but also contributes to ease their geometric representation, which greatly simplifies the optimization process. With this aim, the SBIE and the HBIE are applied separately. Such a simplification of reality is a real asset, especially when compared with the case of the faithful, detailed definition of real complex volumetric designs.

The models studied in this work (Figure 3) have been conveniently designed with the intention of taking advantage of the potential screening properties underlying well-based top geometries. Some of them are inspired on configurations previously studied in the literature. The upper model -model (a)is a QRD-inspired top configuration derived from that studied by Monazzam et al. [22]. Installed on the top of a $0.10 \mathrm{~m}$ width vertical stem, it deals with a $1.00 \mathrm{~m}$ width, $0.30 \mathrm{~m}$ height box comprised of six wells of $0.12 \mathrm{~m}$ width and different depths $\left(d_{i}\right)$ separated by very thin elements. The model in the middle -model (b)- is inspired on the so called waterwheel cylinder studied by Okubo et al. [27]. It is 


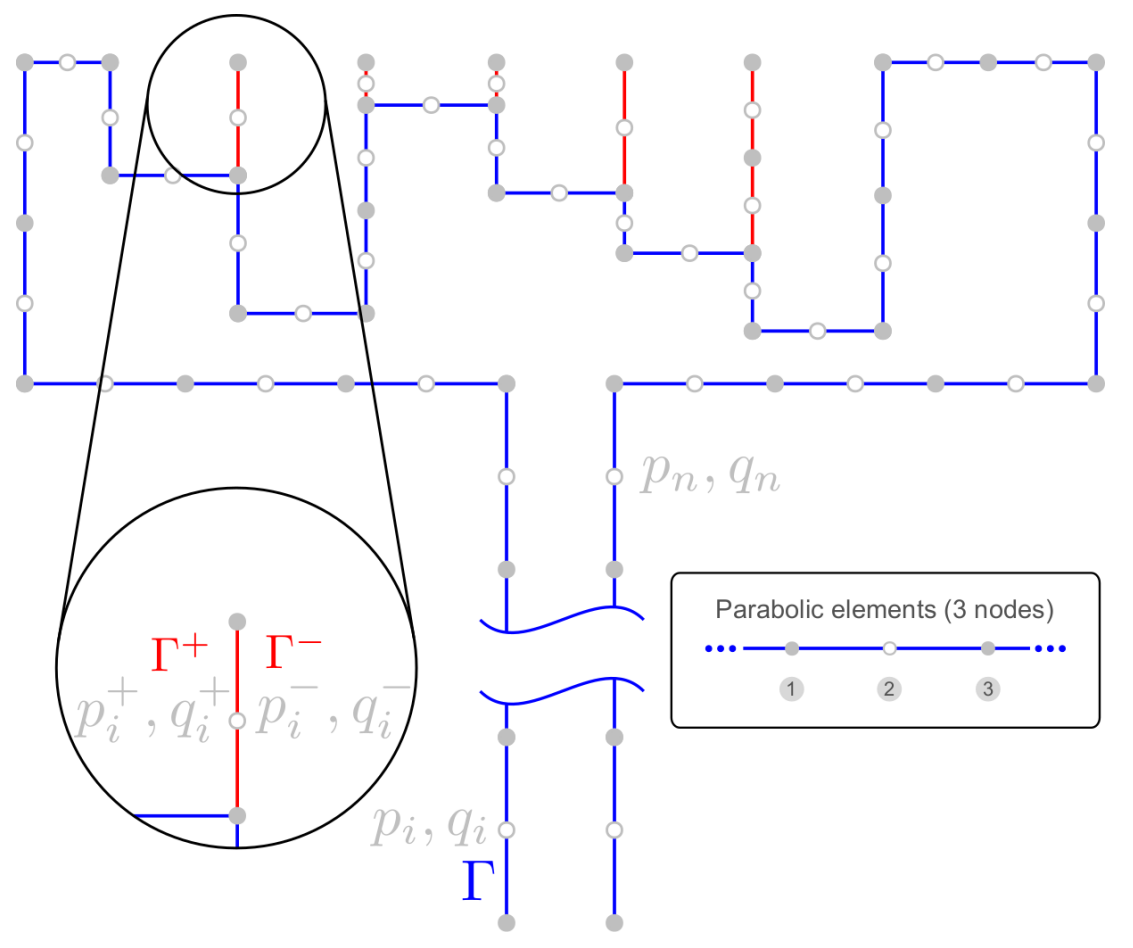

Figure 2: Example of the discretization, after idealization of very thin bodies as null-width elements, with parabolic elements (3 nodes) for $\mathrm{f}=500 \mathrm{~Hz}$ of a QRD-based design. For the ease of viewing, non-thin elements are represented in blue, while very thin bodies are idealized as null-thickness type and depicted in red.

based on a constant-radial top, over a $0.03 \mathrm{~m}$ width vertical stem, comprised of two semi-circular cores with an outer diameter of $0.59 \mathrm{~m}$ from which a uniform distribution of very thin elements are born, for specific well depths $d_{i}$. The model at the bottom -model (c)- is a novel design proposed by the authors of this work. It deals with a top configuration featuring a series of straight and crooked-type wells separated by very thin elements over a $0.10 \mathrm{~m}$ width vertical stem. With the purpose of easing the geometric definition, the very thin elements featuring the top of the models are mathematically considered as null bodies. As in the line of other works by the authors of this paper (e.g., [9 11, 13]), the maximum effective height to be achieved by the barrier is limited to $h_{\text {eff }}=3.0 \mathrm{~m}$ at the median axis. For this reason, despite models (a) and (b) are based on models already studied in the literature, their effective height has been modified to this value.

Generally speaking, the models presented in this work can be categorized as edge-modified barriers, that is, barriers whose top edge has been conveniently shaped with the aim of raising the screening performance of the reference vertical screen. Based on different acoustic mechanisms such as interference and resonance, the adequate shaping of these devices can lead to significantly high noise reduction when compared with the reference barrier for a specific source-receiver scheme [30]. Consequently, despite the apparent design complexity, models similar to those introduced here are designed for practical use [27, 28]. Other interesting devices for practical applications and distributed as commercial 
products in Japan can be consulted in [31].

The shape optimization process aims at searching for series of wells that maximizes the screening performance at the considered receiver point, for both symmetric and non-symmetric configurations of the designs previously described. Models (a) and (b) are comprised of wells with the same potential depth. In both cases, according to the patterns marked by the optimization process, any discrete value from $d_{i}=0.000-0.250 \mathrm{~m}$ (with a discrete step of $0.008 \mathrm{~m}$ ) can be assigned to each well. On the contrary, model (c) has been designed with the purpose of featuring wells with different lengths. In this case, the depth range varies from $d_{i}=0.000-0.185 \mathrm{~m}$ for shortest wells to $d_{i}=0.000-0.808$ $\mathrm{m}$ for longest ones, with discrete step values depending, obviously, on the well length.

Nevertheless, despite the remarkable contribution of some works in the bibliography addressing the acoustic performance of edge-modified barriers with improved designs and others performing the design optimization with GA (that even implement the standard BEM formulation for assessing the shielding behavior), to the authors' knowledge, the procedure here described is the first contribution regarding the efficiency acoustic optimization of top devices coupling a GA with a Dual BEM formulation.

\section{Dual BEM formulation. Acoustic behavior of optimized barrier designs.}

Two are the Dual BEM formulations depending on the nature of the problem under study and the benefit concerning the use of such a strategy. The nature of these problems can be categorized as follows: 1) the mitigation of the fictitious eigenfrequencies; 2) the numerical modeling of thin geometries. Both difficulties have strong presence in the barrier models studied in this work. The features of the formulation for both problems are described in detail in the next subsections.

\subsection{Dual BEM for avoiding fictitious eigenfrequencies}

Some undesirable problems may arise at certain frequencies when dealing with non-thin elements in exterior problems. These mathematically-related effects reveal the eigenfrequencies of the interior acoustic problem (the eigenvalues of the classical BEM matrices) and may seriously affect the intended optimization process. With the purpose of solving this problem, this work makes use of the formulation proposed by Burton and Miller [29] for the exterior problem that features a fictitious resonances-free solution. This formulation is based on the combined use of both the Singular Boundary Integral Equation (SBIE) and the Hyper-singular Boundary Integral Equation (HBIE) coupled by a frequencyrelated complex value $(\alpha)$. The expression for the boundary point $i$ to be solved by BEM can be written then:

$$
0.5\left(p_{i}+\alpha q_{i}\right)+\sum_{j=1}^{N}\left(h_{j}+\alpha m_{j}\right) p_{j}=\sum_{j=1}^{N}\left(g_{j}+\alpha l_{j}\right) q_{j}+\left(G_{0}+\alpha \frac{\partial G_{0}}{\partial n_{i}}\right)
$$

In (11), $p$ is the acoustic pressure field over the barrier surface, $q$ is the flux (the derivative of the pressure with respect to the normal at each boundary node) and $G_{0}$ and $\frac{\partial G_{0}}{\partial n_{i}}\left(H_{0}\right)$ the half-space fundamental solution and its derivative concerning the external noise source, respectively. Finally, $h$ 

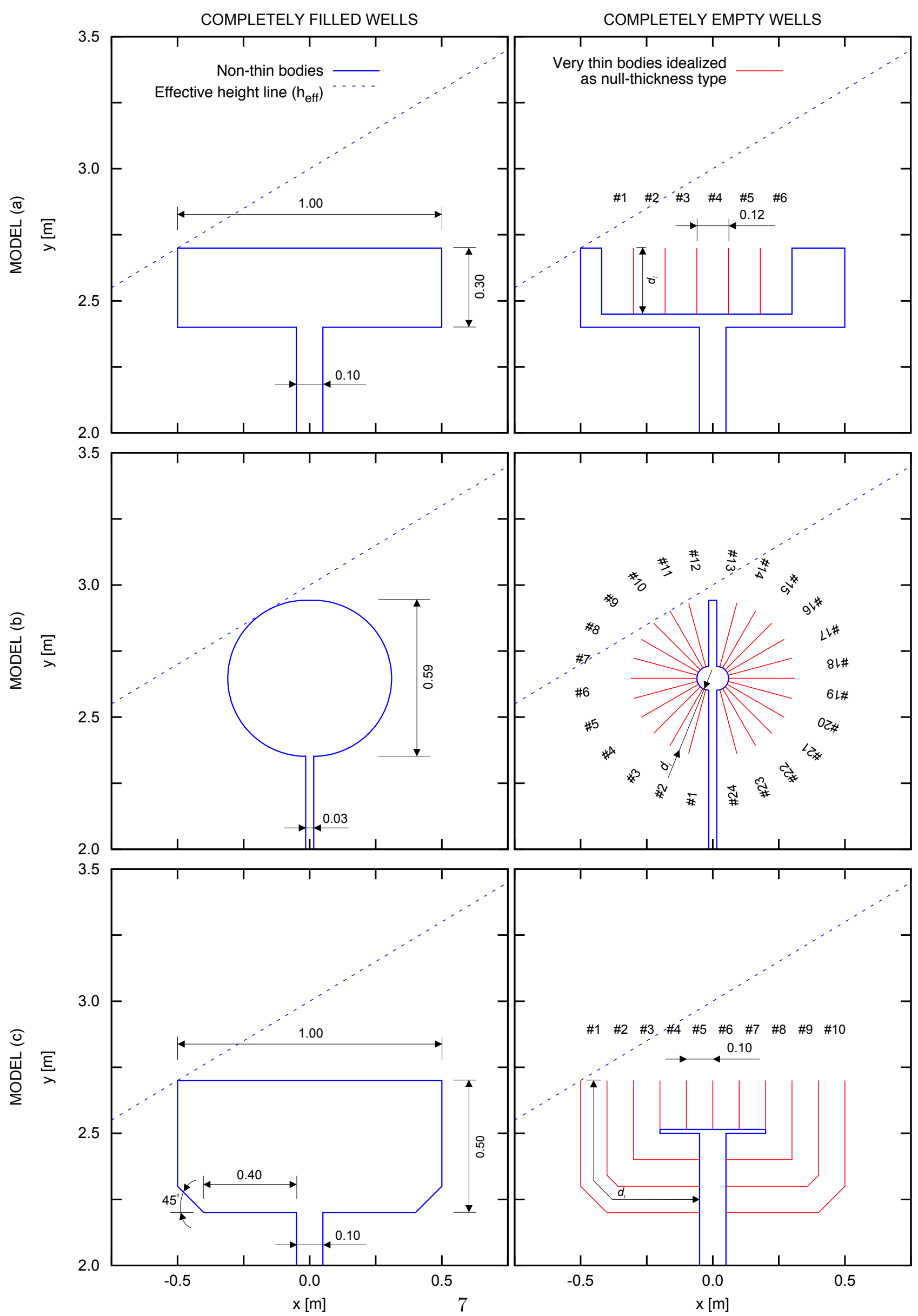

Figure 3: Top designs of the models under study. Left, models featuring just non-thin elements after filling the set of wells $\left(d_{i}=0.000 \mathrm{~m}\right)$. Right, models featuring both non-thin and very thin bodies in the scenario in which every well is completely empty (for models (a) and (b): $d_{i}=0.250 \mathrm{~m}$; for model (c): $d_{1}=d_{10}=0.808 \mathrm{~m}, d_{2}=d_{9}=0.643 \mathrm{~m}, d_{3}=d_{8}=0.350 \mathrm{~m}$ and $\left.d_{4}=d_{5}=d_{6}=d_{7}=0.185 \mathrm{~m}\right)$. For the ease of viewing, non-thin elements are represented in blue, while very thin bodies are idealized as null-thickness type and depicted in red. Dimensions expressed in meters. 
and $g$ are the integration cores of the BEM formulation and $l$ and $m$ the integreation cores of the hiper-singular one, involving just the variables of the problem along the barrier boundary with $N$ nodes after the discretization process. The most commonly used value for the coupling parameter is found to be $\alpha=i / k$ [32, 33], being $i$ the imaginary unit and $k$ the wave number. The hyper-singular formulation of the method demands the collocation point $j$, as known, to be inside the element. This way, the free term is assumed as 0.5 in all cases.

As the barrier boundaries are considered perfectly rigid in this work $\left(q_{j}=0\right)$, (1) can be written matricially:

$$
(0.5 \mathbf{I}+\mathbf{H}+(i / k) \mathbf{M}) \mathbf{P}=\mathbf{G}_{\mathbf{0}}-(i / k) \mathbf{H}_{\mathbf{0}}
$$

with $\mathbf{I}$ being the identity matrix and $\mathbf{H}_{\mathbf{0}}$ a matrix concerning the derivative of the half-space fundamental solution of the external noise source.

\subsection{Dual BEM for very thin bodies}

The nature of the issue is different when dealing with very thin bodies. In this case, numerical integration problems may appear affecting, equally, to the barrier performance. The idealization of such boundaries as non-thickness bodies not only solves the problem but also contributes to ease their geometric representation. With this aim, the SBIE and the HBIE are applied separately ([18, [34]). Figures 2 and 4 facilitates comprehension. The boundaries at both sides of the idealized null-thickness bodies are represented by the discretization, with disparate values of acoustic pressure and flux. The application of the classic formulation of the method, based on the standard boundary integral equation (SBIE) applied at both sides of null-width elements, yields a singular system of equations that does not allow the solution of the problem to be obtained. However, the use of both the SBIE and the HBIE (hyper-singular boundary integral equation) leads to a Dual BEM formulation that offers a proper solution to address problems like the one introduced here.

Figure 4 represents an idealization of a generic thin body to be solved by the Dual BEM formulation. After a discretization process, each node holds the values of pressure and flux with respect to the boundary normal $\left(p^{+}, q^{+}, p^{-}, q^{-}\right.$hereinafter). The strategy used to isolate the singularity of the method in this type of domains can be seen in [35, 36]. Thus, the expression of both the BEM and the hyper-singular BEM formulation for these boundaries can be written as follows:

$$
\begin{gathered}
0.5\left(p_{i}^{+}+p_{i}^{-}\right)+\sum_{j=1}^{N}\left(H_{j}^{+} p_{j}^{+}+H_{j}^{-} p_{j}^{-}\right)=\sum_{j=1}^{N}\left(G_{j}^{+} q_{j}^{+}+G_{j}^{-} q_{j}^{-}\right)+G_{0}(k, r) \\
0.5\left(\frac{\partial p_{i}^{+}}{\partial n_{i}^{+}}+\frac{\partial p_{i}^{-}}{\partial n_{i}^{+}}\right)+\sum_{j=1}^{N}\left(M_{j}^{+} p_{j}^{+}+M_{j}^{-} p_{j}^{-}\right)=\sum_{j=1}^{N}\left(L_{j}^{+} q_{j}^{+}+L_{j}^{-} q_{j}^{-}\right)
\end{gathered}
$$

being $N$ the overall nodes number of the discretization over the boundary. Taking into account that $n^{+}=-n^{-}$, it is easily shown that:

$$
\begin{gathered}
H_{j}^{+}=-H_{j}^{-} \quad ; \quad G_{j}^{+}=G_{j}^{-} \\
\frac{\partial p_{i}^{-}}{\partial n_{i}^{+}}=-q_{i} \quad ; \quad M_{j}^{+}=-M_{j}^{-} \quad ; \quad L_{j}^{+}=L_{j}^{-}
\end{gathered}
$$




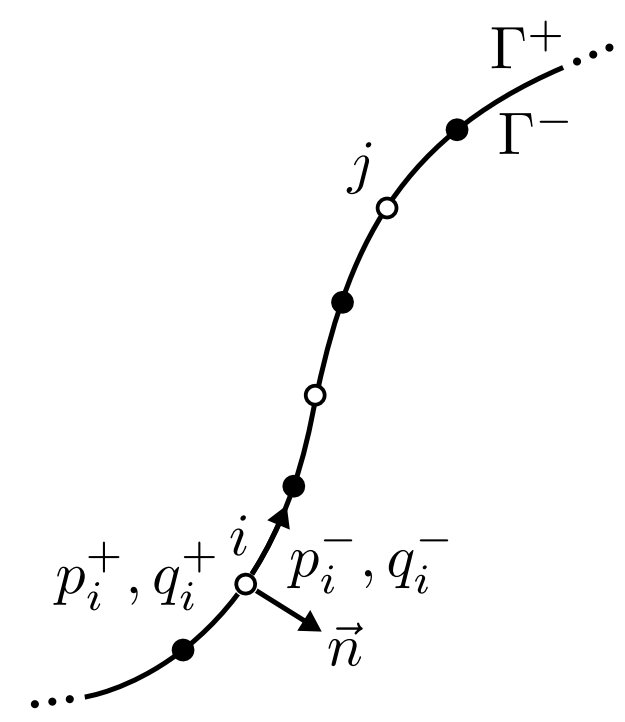

Figure 4: Idealization of a generic thin-cross section noise barrier profile as null thickness boundaries. Discretization with parabolic elements (3 nodes).

As before and taking into consideration that barrier boundaries are considered perfectly rigid in this work $\left(q_{j}=0\right)$, the following matrix expressions are obtained:

$$
\left(0.5 \mathbf{I}^{*}+\mathbf{H}\right) \mathbf{P}=\mathbf{G}_{0}
$$

$$
\mathbf{M} \cdot \mathbf{P}=\mathbf{H}_{\mathbf{0}}
$$

being $\mathbf{I}^{*}$ a matrix that allows us to consider the contribution of the free term at both sides of the discretization nodes and $\mathbf{H}_{\mathbf{0}}$ a matrix concerning the derivative of the half-space fundamental solution of the external noise source.

The Dual BEM model used to address the problems under study in this work incorporates, as previously mentioned, both versions of the dual formulation. To enable an easier, more general procedure, a multiple collocation of nodes at extremes of elements with different nature (that is, at borders between thin and non-thin bodies) is implemented. Once the variables on the barrier boundary are known, the acoustic pressure values at any internal point (receiver position) can be easily obtained, as usual, by applying the standard BEM formulation.

The numerical aspects related to the evaluation of both the singular and the hyper-singular BEM integrals developed and implemented in the computer code can be consulted in [35, 37-39].

The validation of the Dual BEM code is performed on the basis of results present in the reference papers from which model (a) and (b) (derived from a QRD and the so called waterwheel cylinder, respectively) are inspired $([22,27])$. 


\section{Shape optimization}

Figure 5 represents the general configuration for just one of the models under study -model (a)-. It deals with a two-dimensional model concerning an infinite, coherent mono-frequency source of sound, generating pure tones within the considered frequency spectrum, parallel to an infinite barrier with no geometric variation that stands on a flat plane (ground) of uniform admittance. Both the ground and the barrier boundary feature a perfectly reflecting surface $\left(\beta_{b}=\beta_{g}=0\right)$. Just one receiver in the shadow region is considered in assessing the overall acoustic efficiency (to be maximized along the optimization process). Both the noise source and the receiver are located on the ground at a horizontal distance of 5.0 and $25.0 \mathrm{~m}$, respectively. As stated above, the maximum effective height to be achieved is $h_{\text {eff }}=3.0 \mathrm{~m}$ at the median vertical axis of the barrier.

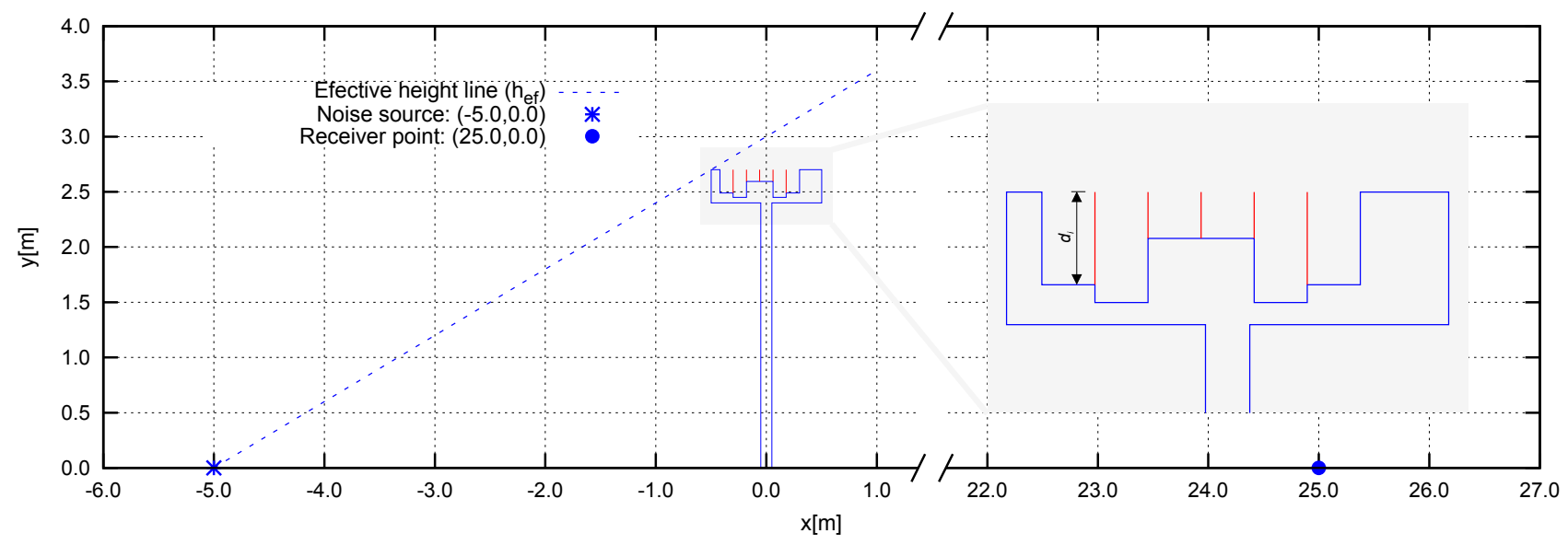

Figure 5: Bi-dimensional configuration to be used in the optimization process of the models presented in this work. Example of a QRD-inspired top design (model (a) in this paper) with different well depths $d_{i}$.

Shape design optimization is performed by the combined use of an evolutionary algorithm and the mentioned Dual BEM formulation. The evolutionary algorithm software used in this work applies the GAlib package [40]. This library is a collection of $\mathrm{C}++$ genetic algorithm (GA) components from which it is possible to quickly construct GA's to attack a wide variety of problems.

In the harmonic problem, for every frequency from the analyzed noise source, the effectiveness of the barrier design under study is given in terms of the insertion loss (IL), defined as usual:

$$
\mathrm{IL}=-20 \cdot \log _{10}\left(\frac{\mathrm{P}_{\mathrm{B}}}{\mathrm{P}_{\mathrm{HS}}}\right)[\mathrm{dB}]
$$

on every frequency of the broadband spectrum, and represents the difference of sound pressure levels at the receiver point in the situation with $\left(\mathrm{P}_{\mathrm{B}}\right)$ and without $\left(\mathrm{P}_{\mathrm{HS}}\right)$ considering the barrier.

With the purpose of conducting an optimization process where the excitation is represented by a noise source pulsing at every frequency of the band spectrum, the efficiency of the barrier for the 
Table 1: Description of the design variables of each model (well length, coded with binary bits) and of the corresponding chromosome.

\begin{tabular}{|c|c|c|c|c|}
\hline Model & $\begin{array}{l}\text { Chromosome } \\
\text { length }\left(\mathrm{n}_{\mathrm{ch}}\right)\end{array}$ & $\begin{array}{l}\text { Bit precision } \\
\text { per variable }\end{array}$ & $\begin{array}{c}\text { Design variable } \\
\text { range }[\mathrm{m}]\end{array}$ & $\begin{array}{c}\text { Discrete values } \\
\text { per variable }\end{array}$ \\
\hline \multirow{2}{*}{ (a) } & Symmetric: 15 & \multirow{4}{*}{5 (each well) } & \multirow{4}{*}{$0.000-0.250$} & \multirow{4}{*}{32} \\
\hline & Non-symmetric: 30 & & & \\
\hline \multirow{2}{*}{ (b) } & Symmetric: 60 & & & \\
\hline & Non-Symmetric: 120 & & & \\
\hline \multirow{4}{*}{ (c) } & & \multirow{4}{*}{$\begin{array}{l}\text { Well \#1: } 5 \\
\text { Well \#2: } 5 \\
\text { Well \#3: } 5 \\
\text { Well \#4: } 3 \\
\text { Well \#5: } 3\end{array}$} & $0.000-0.808$ & \multirow{3}{*}{32} \\
\hline & Symmetric: 21 & & $0.000-0.643$ & \\
\hline & \multirow[b]{2}{*}{ Non-symmetric: 42} & & $0.000-0.350$ & \\
\hline & & & $0.000-0.185$ & 8 \\
\hline
\end{tabular}

considered receiver can be written in terms of the broadband insertion loss as:

$$
\mathrm{IL}_{\text {total }}=-10 \cdot \log _{10}\left(\frac{\sum_{\mathrm{i}=1}^{\mathrm{NF}} 10^{\left(\mathrm{A}_{\mathrm{i}}-\mathrm{IL}_{\mathrm{i}}\right) / 10}}{\sum_{\mathrm{i}=1}^{\mathrm{NF}} 10^{\mathrm{A}_{\mathrm{i}} / 10}}\right)[\mathrm{dBA}]
$$

being NF the studied spectrum number of frequencies, $A_{i}$ the spectrum A-weighted noise level and $\mathrm{IL}_{\mathrm{i}}$ the insertion loss value for sources pulsing at every frequency of the spectrum, according to (9).

In this work, the noise source has been characterized by using the UNE-EN 1793 [41] normalized traffic noise spectrum, generating pure tones ranging from 100 to $5000 \mathrm{~Hz}$ corresponding to one-third center band frequencies. Given the high frequency dependence of the studied designs and to assess as accurately as possible the broadband IL, the $1 / 3$-octave bands are expanded to $1 / 15$ octave intervals, represented by band center frequencies. The normalized levels for $1 / 15$ octave frequency bands in this corrected spectrum are calculated in such a way that the total acoustic intensity is the same than that of the original. The estimator taken into account along the shape optimization process, that is, the objective function, is entirely based on the IL average spectrum value at the considered receiver point $\left(\mathrm{OF}=\mathrm{IL}_{\text {total }}\right)$. The maximization of this latter parameter through the optimization process is intended.

As each objective function evaluation requires the execution of a high cost CPU for BEM, a high exploitative strategy with high selection pressure has been taken into account: a steady-state genetic algorithm [42, 43] is used replacing the two worst individuals (in terms of their objective function) at each generation. A population size of 100 individuals, with a two-point crossover operator (crossover rate equal to 0.9 ) is used in this study. The considered mutation rate is $1 / \mathrm{n}_{\mathrm{ch}}$, where $\mathrm{n}_{\mathrm{ch}}$ is the chromosome length with design variables coded with different binary bit precision, depending on the model, according to the Gray code (see Table 1). Five independent runs of the optimization process are considered for each symmetric and non-symmetric design. The stopping criterion condition is met for 1000 generations. 
To facilitate understanding of the methodology, Figure 6 shows a flow diagram concerning the evolutionary process on the search of the best acoustic solutions. The procedure makes use of a steady state GA with individuals initially based on a proposal on random design variables of the model under assessment, featured by discrete values of well length. A detailed description concerning the definition of both the design variables and the chromosome of each model is collected in Table1, Such design variables form the chromosome of the individual proposed by the GA shaping, therefore, the geometry of the top device of the barrier. At this point, the screening behavior of each individual is performed using the aforementioned Dual BEM code. This requires a proper discretization of the barrier boundary with ever-increasing number of elements along the frequency spectrum. In this work, parabolic elements are coded with a maximum length of half the wavelength of the frequency under study for adequate convergence of the results. The initial population is then ranked in terms of acoustic performance, here represented by the objective function value (OF) characterized, in turn, by the broadband insertion loss $\left(\mathrm{IL}_{\text {total }}\right)$ (see (10)). In this way, each individual is more likely to be selected according to its screening behavior (OF). Therefore, by using the tournament selection operator, two individuals are chosen (parents in proper terminology of evolutionary algorithms) and are to be crossed with a probability of $90 \%$ by the two-point crossover operator, leading to an offspring comprised of two new individuals (children) whose chromosomes are eligible for mutation according to probabilistic criteria. After mutation, offspring individuals replace the two worst in the parent population (in case of better value of $\mathrm{OF}$, that is, improvement of the acoustic behavior). This iterative process continues until reaching the stopping criterion, which is set at 1000 generations due to balancing an acceptable convergence of the results with invested computational resources.

\section{Results}

\subsection{Designs after shape optimization}

Figure 7 illustrates the results concerning the best optimum individuals from the optimization processes. In the left part, the top geometries of each model for symmetric and non-symmetric configurations are shown. The right part depicts the IL spectrum evolution of such optimum designs along the considered frequency range, in comparison with their corresponding model for both completely filled and completely empty wells (see Figure 31) and with a $3 \mathrm{~m}$ vertical screen, for the given noise source-receiver scheme. The overall acoustic performance of the optimum designs, according to (10), is also included in the figure (see graphic legend). In addition, Figure 8 shows the acoustic performance evolution of the presented models along the optimization process. Red lines represent the shielding efficiency of the best individual found at each generation, in terms of the objective function (OF), within the 5 runs considered. Blue lines depict the OF mean value considering the best individual of each run (5 individuals in all) at each generation. As observed, the graphs are shown in an adjusted range in the ordinate coordinate. This facilitates viewing of the results with no loss of relevant details. The results achieved suggest that further evolution is needed for a proper convergence, specially in the case of non-symmetric configurations and, in particular, for model (b). The symmetric configuration of model (c) and both configurations of model (a) present an acceptable convergence, though. Numerical results concerning this graph can be consulted in Table 2.

Table 3 presents the well depths $\left(d_{i}\right)$ of the best optimum designs for each model, configuration and 
run. As an example, the corresponding computing time for model (a) cases is 97 hours on average, run in a CPU Intel ${ }^{\circledR}$ Xeon ${ }^{\circledR} 2.60 \mathrm{GHz}$ processor (RAM is not significant here). Row $\Delta \mathrm{IL}_{\text {total }}^{3 \mathrm{~m} \text { vert. collects }}$ the acoustic efficiency gain of such optimums in comparison with the reference $3 \mathrm{~m}$ vertical barrier.

Finally, with the intention of illustrating the effects of the best optimum designs of non-symmetric configurations, Figure 9 shows in colormaps the broadband sound pressure level SPL $_{\text {total }}$, expressed in $\mathrm{dBA}$ ) in a domain with a noise source intensity of $90 \mathrm{dBA}$ (measured $1 \mathrm{~m}$ away in the free field) pulsing at every frequency according to the spectrum considered so far. Results are easily obtained through simple operations from the $\mathrm{IL}_{\text {total }}$ values associated with such optimums.

Table 2: Average and best values of all runs of the objective function (OF) for the considered configurations and models.

\begin{tabular}{lrcc} 
& & Best OF & Average OF \\
\cline { 3 - 4 } Model (a) & Symmetric & 16.99 & 16.97 \\
& Non-symmetric & 17.13 & 17.09 \\
\hline \multirow{2}{*}{ Model (b) } & Symmetric & 16.62 & 16.60 \\
& Non-symmetric & 16.62 & 16.59 \\
\hline \multirow{2}{*}{ Model (c) } & Symmetric & 18.75 & 18.74 \\
& Non-symmetric & 19.03 & 19.01 \\
\hline
\end{tabular}


Table 3: Design variables $d_{i}$ (in centimeters) of the best optimum barrier profiles (see Figure 7) and shielding efficiency gain with respect to a $3 \mathrm{~m}$ vertical screen (in dBA) for each model and configuration.

\begin{tabular}{|c|c|c|c|c|c|c|}
\hline & \multicolumn{3}{|c|}{ Symmetric } & \multicolumn{3}{|c|}{ Non-symmetric } \\
\hline & $\overline{\text { Model (a) }}$ & Model (b) & $\overline{\text { Model }(\mathrm{c})}$ & $\overline{\text { Model (a) }}$ & Model (b) & Model (c) \\
\hline$d_{1}$ & 11.29 & 0.00 & 44.34 & 11.29 & 1.61 & 75.64 \\
\hline$d_{2}$ & 25.00 & 1.61 & 53.92 & 25.00 & 1.61 & 51.85 \\
\hline$d_{3}$ & 21.77 & 5.65 & 32.74 & 20.97 & 8.87 & 31.61 \\
\hline$d_{4}$ & 21.77 & 17.74 & 18.50 & 17.74 & 20.16 & 10.57 \\
\hline$d_{5}$ & 25.00 & 25.00 & 18.50 & 25.00 & 23.39 & 18.50 \\
\hline$d_{6}$ & 11.29 & 23.39 & 18.50 & 25.00 & 22.58 & 18.50 \\
\hline$d_{7}$ & - & 24.19 & 32.74 & - & 24.19 & 18.50 \\
\hline$d_{8}$ & - & 22.58 & 53.92 & - & 23.39 & 22.58 \\
\hline$d_{9}$ & - & 18.55 & 44.34 & - & 22.58 & 60.14 \\
\hline$d_{10}$ & - & 15.32 & - & - & 12.10 & 41.73 \\
\hline$d_{11}$ & - & 12.10 & - & - & 0.00 & - \\
\hline$d_{12}$ & - & 0.00 & - & - & 0.81 & - \\
\hline$d_{13}$ & - & 0.00 & - & - & 10.48 & - \\
\hline$d_{14}$ & - & 12.10 & - & - & 17.74 & - \\
\hline$d_{15}$ & - & 15.32 & - & - & 16.94 & - \\
\hline$d_{16}$ & - & 18.55 & - & - & 23.39 & - \\
\hline$d_{17}$ & - & 22.58 & - & - & 22.58 & - \\
\hline$d_{18}$ & - & 24.19 & - & - & 25.00 & - \\
\hline$d_{19}$ & - & 23.39 & - & - & 24.19 & - \\
\hline$d_{20}$ & - & 25.00 & - & - & 21.77 & - \\
\hline$d_{21}$ & - & 17.74 & - & - & 12.10 & - \\
\hline$d_{22}$ & - & 5.65 & - & - & 1.61 & - \\
\hline$d_{23}$ & - & 1.61 & - & - & 1.61 & - \\
\hline$d_{24}$ & - & 0.00 & - & - & 1.61 & - \\
\hline$\Delta \mathrm{IL}_{\text {total }}^{3 \mathrm{~m} \text { vert. }}$ & +3.06 & +2.67 & +4.82 & +3.20 & +2.68 & +5.10 \\
\hline
\end{tabular}



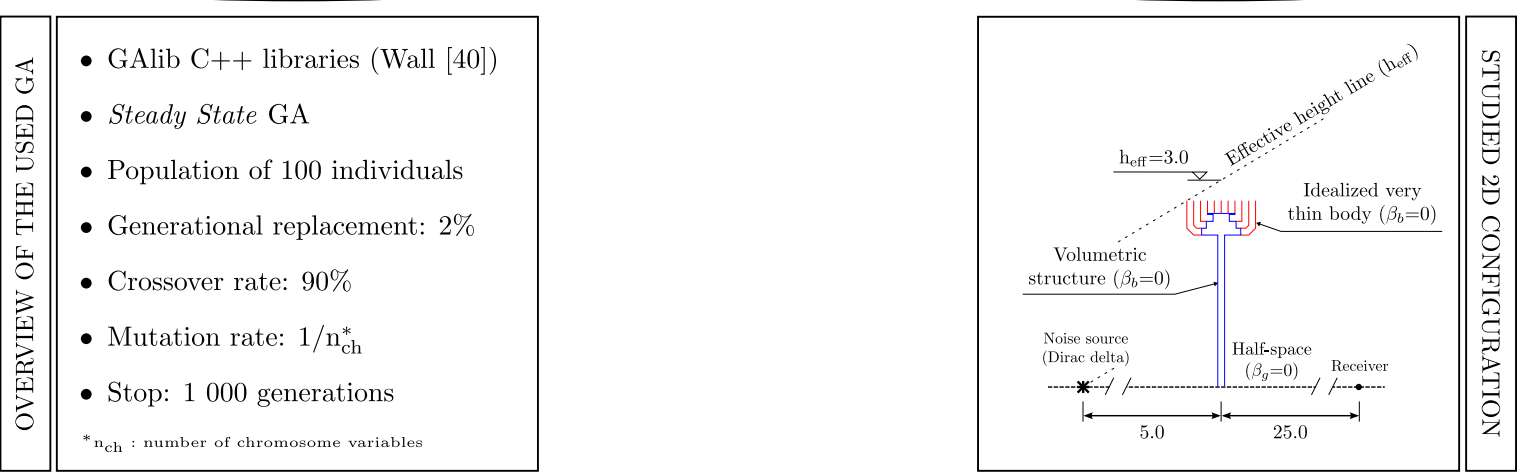

\section{EVOLUTIONARY PROCESS}

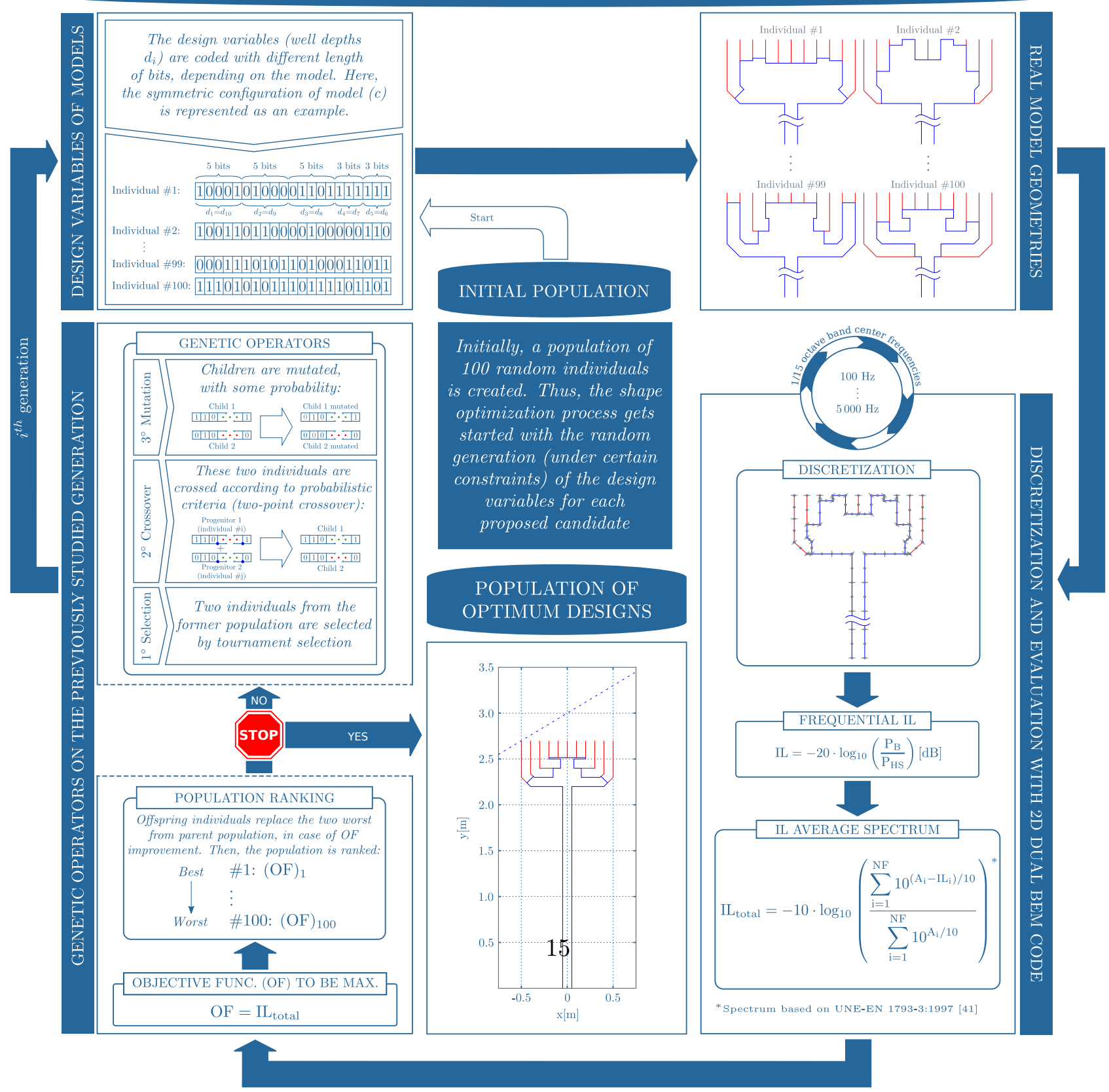

Figure 6: Overview of the GA used in this work, layout of the considered bi-dimensional configuration and optimization flow diagram. 


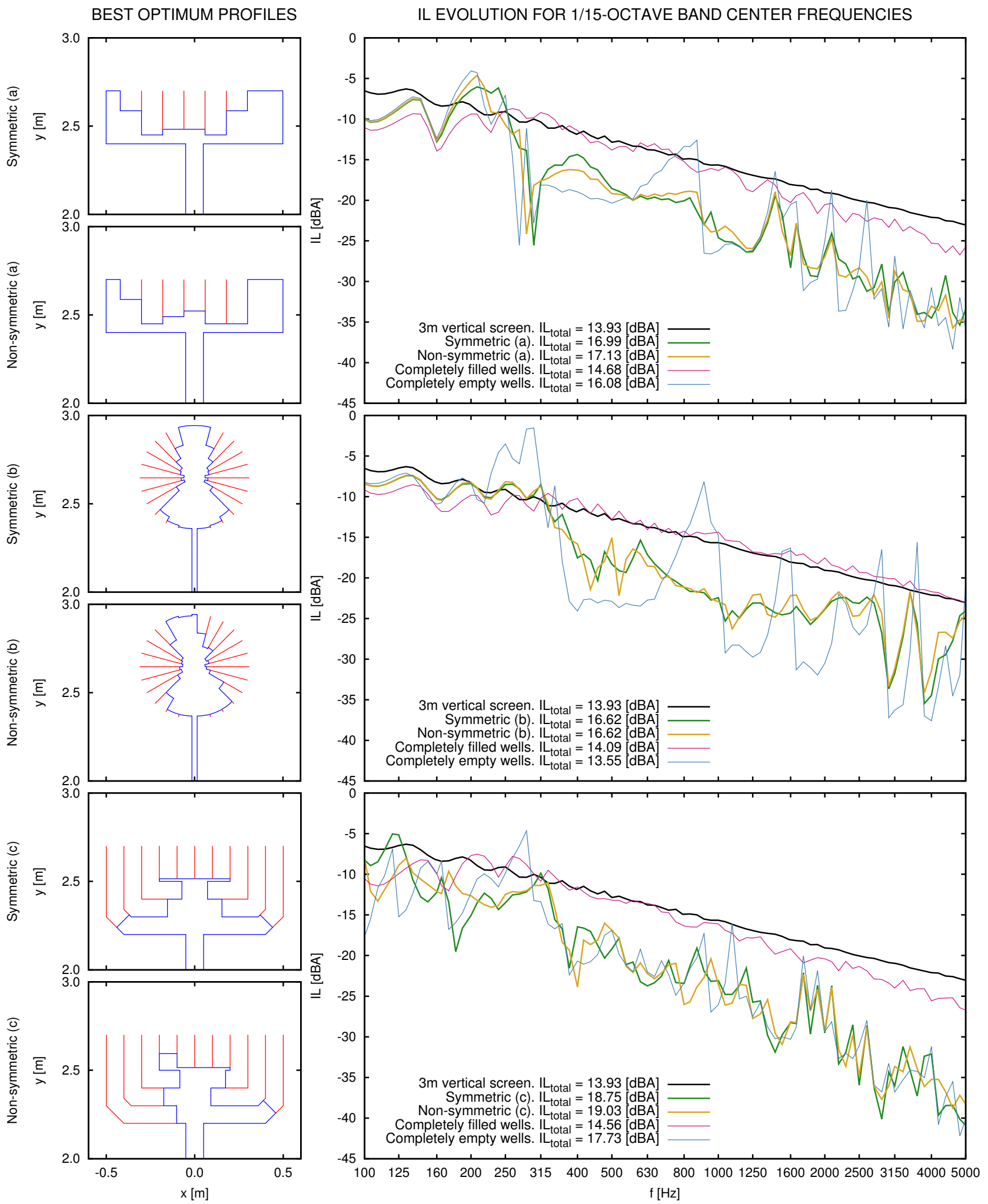

Figure 7: Results for the best individuals found along the optimization processes for each model and configuration. Left, best optimum profiles. Right 6 IL along the considered $1 / 15$-octave band center frequencies for the aforementioned geometries and their corresponding model for both completely filled and completely empty wells (see Figure 3). 

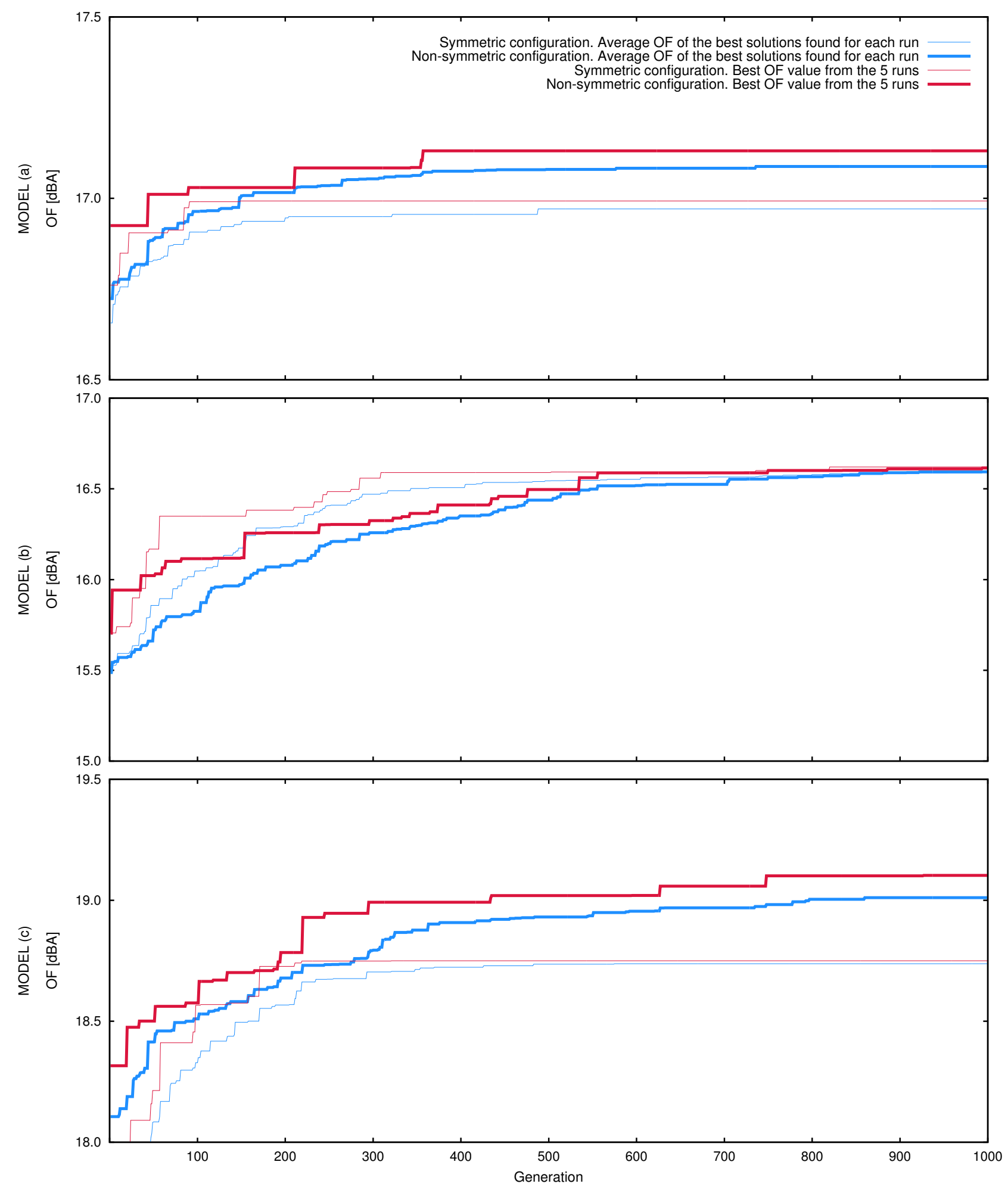

Figure 8: Objective function (OF) convergence charts for both symmetric and non-symmetric configurations of the studied models. 

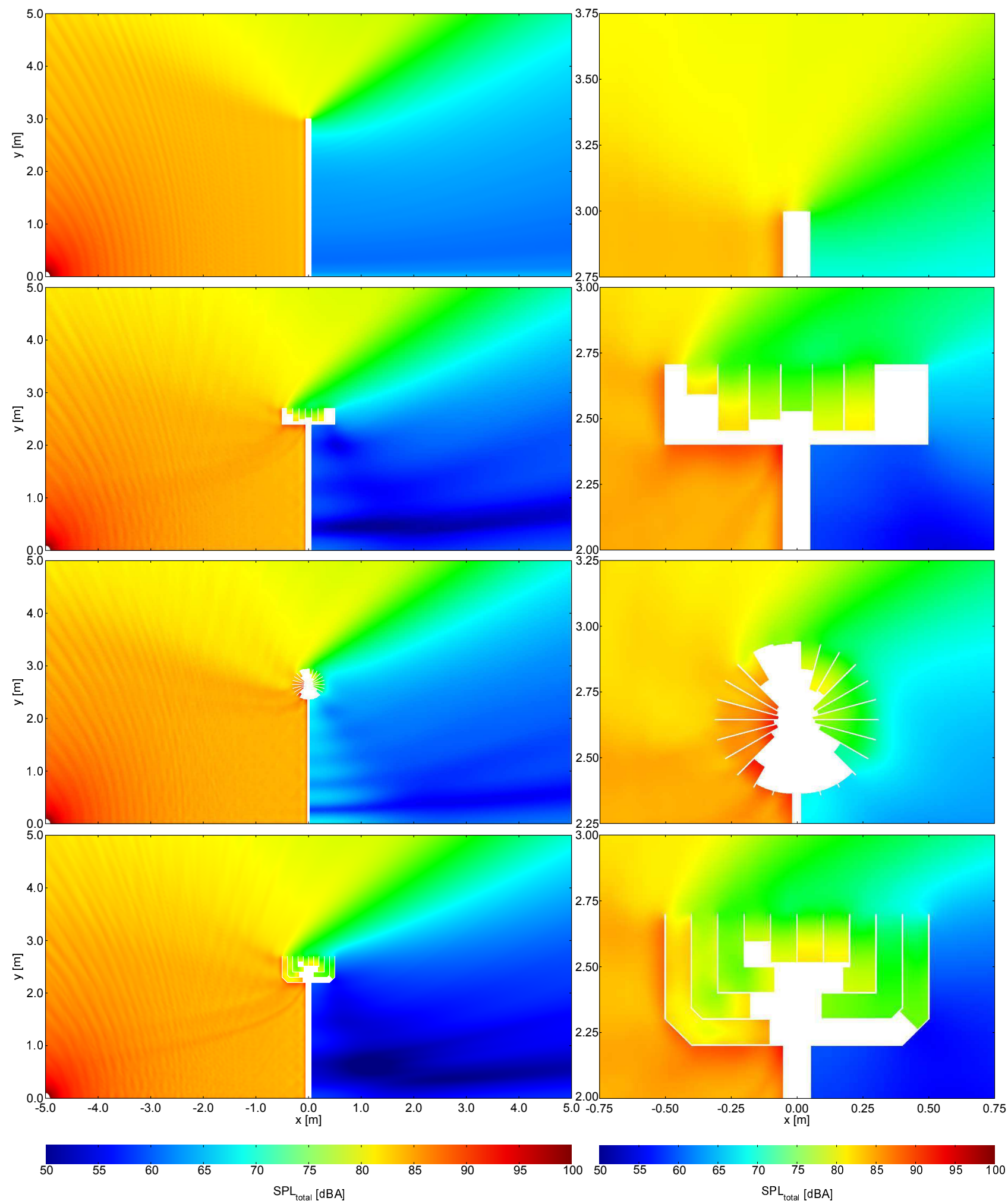

Figure 9: Broadband sound pressure level ( $\left.\mathrm{SPL}_{\text {total }}\right)$ colormaps for a sound intensity level of $90 \mathrm{dBA}$

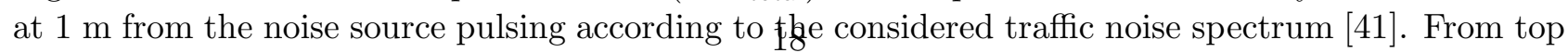
to bottom, results for a $3 \mathrm{~m}$ vertical screen and best optimum non-symmetric designs for model (a), model (b) and model (c). Noise source at $(-5.0,0.0)$. 


\subsection{Designs for practical use}

With the intention of both determining how important the role of wells at the top is in the shielding behavior and leading to easier-to-build, more practical barrier designs, Figure 10 shows some geometric modifications on the basis of the optimum symmetric and non-symmetric configuration of model (b). Starting off from such designs after the GA optimization, slight modifications, in terms of emptyingfilling patterns of some wells, are introduced (represented by Mod. \#1 to Mod. \#7). In brief, three sets of wells with the same depth are considered, represented by the wells at the bottom, at the middle and at the top of the device. In addition, a design previously studied in the literature by Okubo et al. 27] is included as reference. Results are presented in terms of the broadband sound pressure level $\left(\mathrm{SPL}_{\text {total }}\right)$ in the vicinity of a noise source pulsing with a sound intensity level of $90 \mathrm{dBA}$ according to the used traffic noise spectrum [41] for the considered source-receiver scheme. This way, the lower the $\mathrm{SPL}_{\text {global }}$, the better. As observed, the practical designs outperform the reference case over a decibel in most cases for the symmetric configuration.

On the other hand, the benefits of the methodology introduced in this work are shown in Figure11. As observed, the acoustic performance of the best optimum designs for symmetric and non-symmetric configurations of model (a) are compared with a QRD of same topological design, previously studied in the literature by Monazzam et al. [22]. Results suggest the convenience of implementing procedures like that presented here for raising the acoustic performance of already existing barrier designs. 


\begin{tabular}{|c|c|c|c|c|c|c|c|c|}
\hline $\begin{array}{l}\text { Optimum } \\
\text { symmetric }\end{array}$ & Mod. \#1 & Mod. \#2 & Mod. \#3 & Mod. \#4 & Mod. \#5 & Mod. \#6 & Mod. \#7 & Okubo et al. [27] \\
\hline$S P L_{\text {total }}=58.61$ & $S P L_{\text {total }}=58.75$ & $S P L_{\text {total }}=59.00$ & $\mathrm{SPL}_{\text {total }}=59.65$ & $S P L_{\text {total }}=60.06$ & $\mathrm{SPL}_{\text {total }}=60.80$ & $\mathrm{SPL}_{\text {total }}=59.56$ & $S P L_{\text {total }}=59.99$ & $S P L_{\text {total }}=61.69$ \\
\hline
\end{tabular}
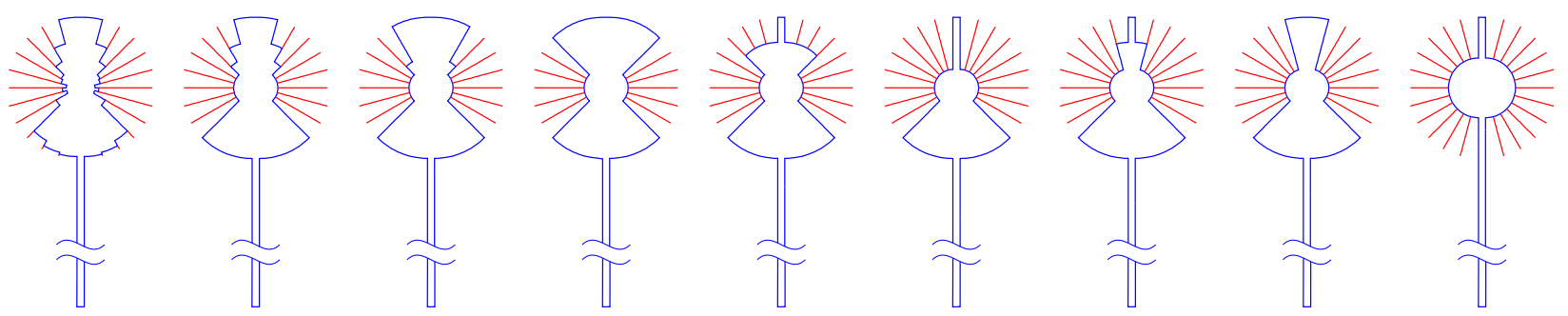

Optimum non-symmetric $\mathrm{SPL}_{\text {total }}=58.62$

$\mathrm{SPL}_{\text {total }}=59.03 \mathrm{SP}$
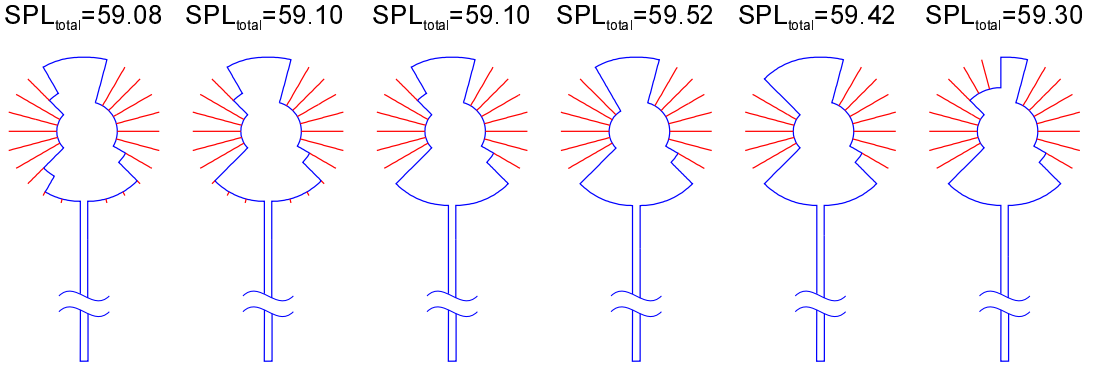

Reference

$\mathrm{SPL}_{\text {total }}=61.64$

Figure 10: Slight geometry modifications on the basis of reference optimum designs of model (b): up, modifications from the best optimum symmetric configuration; bottom, modifications from the best optimum non-symmetric configuration. Results expressed in terms of the broadband sound pressure level $\left(\mathrm{SPL}_{\text {total }}\right)$ measured in the considered receiver point in $\mathrm{dBA}$, for a scheme featuring a noise source intensity of $90 \mathrm{dBA}$ (measured $1 \mathrm{~m}$ away in the free field) pulsing at every frequency according to the considered spectrum.

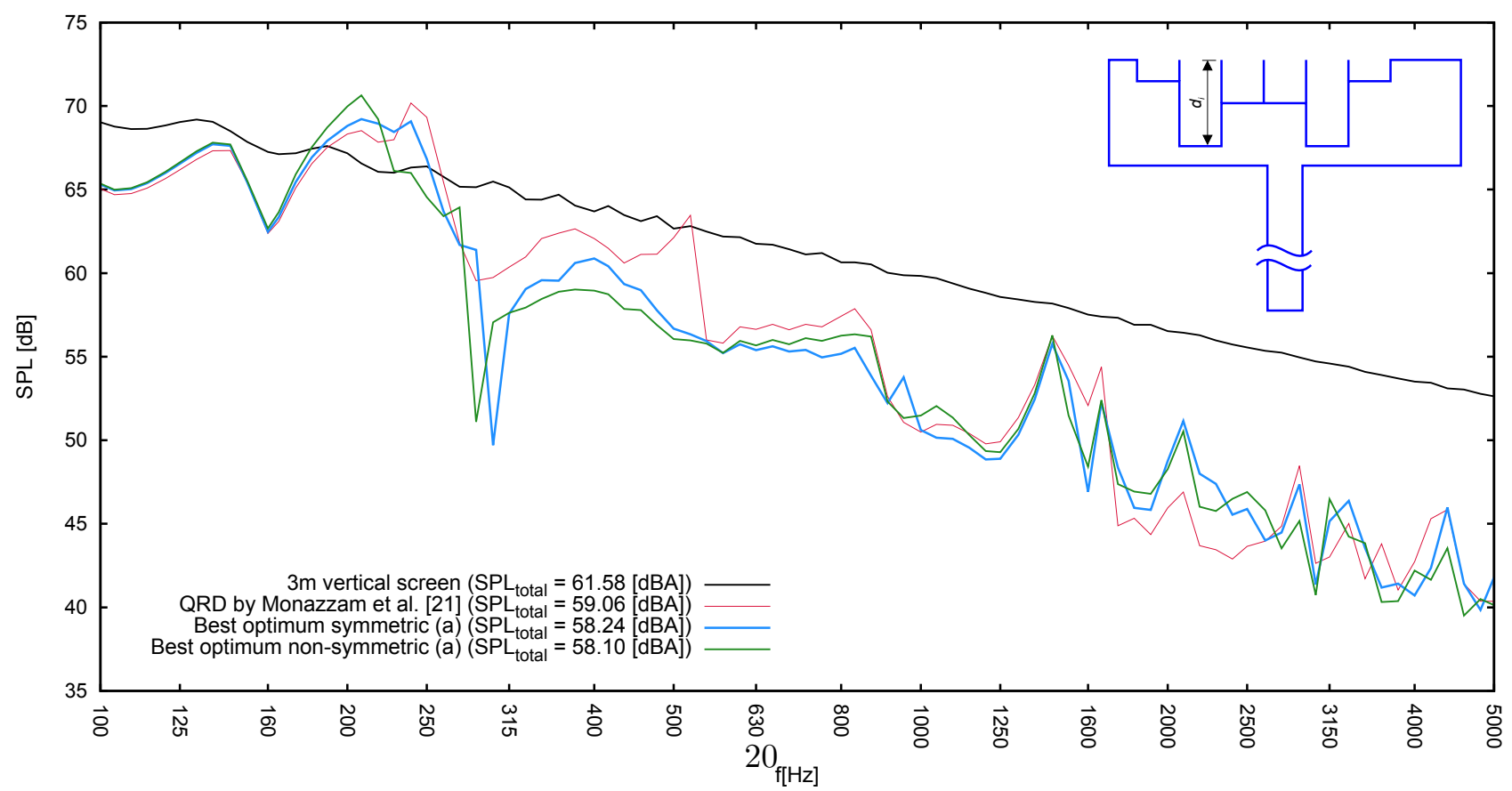

Figure 11: Sound pressure level (SPL) evolution. Graphs for a QRD $\left(d_{1}=d_{6}=0.0600 \mathrm{~m}, d_{2}=\right.$ $d_{5}=0.24450 \mathrm{~m}, d_{3}=d_{4}=0.12225 \mathrm{~m}$ ), the best optimum designs for symmetric and non-symmetric configurations of model (a) ( $d_{i}$ in Table 3) and a $3 \mathrm{~m}$ vertical screen. 


\subsection{Discussion}

From the analysis of the results obtained some conclusions on the response of the models studied in this work may be drawn:

- The shielding efficiency of the models studied here clearly outperform the acoustic behavior of the reference $3 \mathrm{~m}$ vertical screen for the considered noise source-receiver scheme. The non-so-near placement of the receiver point makes this issue more remarkable.

- The use of designs with longer wells raises the acoustic performance of the barrier. This is well illustrated by model (c) (Figure 7), where the IL curve performs far better than the $3 \mathrm{~m}$ vertical screen even for low frequencies.

- As expected, generally speaking, non-symmetric best designs are always better than symmetric ones, both in best values and average values. Even in the model (b) case, the non-symmetric optimum designs could have far outperformed the symmetric ones in a hypothetical scenario with higher number of generations (as seen in the convergence evolution of the search in Figure 8).

- Despite the well known strong frequency dependence-nature of these well-shaped devices (e.g., [27]), the optimum profiles present smoother IL curves than their corresponding model featuring completely empty wells (see Figure 7) but with considerably higher shielding efficiency.

- As shown in Figure 8, a slightly further convergence of the acoustic performance of the studied models (particularly of models (b) and (c)) is still expected. Constraints regarding the considered spectrum (1/15-octave bands center frequencies) impose a high consuming CPU time per objective function evaluation, especially at high frequencies (the higher the frequency, the finer the required BEM mesh). However, the stopping criterion adopted in this work seems to have achieved reasonable convergence for the limited invested computational resources.

- The consideration of methodologies like the one here presented allows the search of interesting shielding solutions to be easily accomplished and can be considered as an adequate procedure to outperform the screening behavior of already existing barrier designs (see Figures 10 and 11).

\section{Conclusions}

A procedure for the shape optimization of well-based designs on the top of road barriers with both thick and very thin bodies by coupling a genetic algorithm with a 2D Dual BEM code has been presented. In order to ease the geometric definition of such designs, the very thin elements featuring the top of the barriers have been mathematically considered as null bodies. The methodology presented in this work focuses on searching for series of wells based on discrete values that maximizes the screening performance of the aforementioned models. With the purpose of highlighting the robustness and flexibility of the methodology, numerical results of three complex designs on the top of road barriers for both symmetric and non-symmetric configurations have been presented. The results show that despite the well known strong frequency dependence-nature of these well-shaped devices, the optimum profiles present smoother IL curves than their corresponding model featuring completely empty wells but with considerably higher shielding efficiency. In addition, the use of designs with potential deeper 
wells considerably raises the acoustic performance of the barrier since the shielding efficiency performs well from low frequencies onwards, for the considered source-receiver scheme.

The versatility of the algorithm responsible for the geometry generation of the barrier makes the building of the profile to be easily accomplished. The Dual Boundary Element formulation here presented allows a simple treatment of the complex configurations. This is a significant advantage over the case when dealing with geometries of real barrier profiles, as the evaluation process for the feasibility of the design proposed by an evolutionary algorithm is often cumbersome and difficult to establish.

\section{Acknowledgment}

This work was supported by the Ministerio de Economía y Competitividad (MINECO) of Spain and by Fondo Europeo de Desarrollo Regional (FEDER) through research project BIA2010-21399-C02-01. R. Toledo is a recipient of a fellowship from the Subprogram of Predoctoral Fellowships of Research Personnel in Training (FPI-MICINN), granted by Ministerio de Ciencia e Innovación of Spain. The authors are grateful for this support.

\section{References}

[1] R. Seznec, Diffraction of sound barrires: use of the boundary element technique, Journal of Sound and Vibration 73 (1980) 195-209.

[2] D. C. Hothersall, S. N. Chandler-Wilde, M. N. Hajmirzae, Efficiency of single noise barriers, Journal of Sound and Vibration 146 (1991) 303-322.

[3] D. C. Hothersall, D. H. Crombie, S. N. Chandler-Wilde, The performance of T-profile and associated noise barriers, Applied Acoustics 32 (1991) 269-287.

[4] D. H. Crombie, D. C. Hothersall, The performance of multiple noise barriers, Journal of Sound and Vibration 176 (1994) 459-73.

[5] D. H. Crombie, D. C. Hothersall, S. N. Chandler-Wilde, Multiple-edge noise barriers, Applied Acoustics 44 (1995) 353-367.

[6] G. R. Watts, S. N. Chandler-Wilde, P. A. Morgan, The combined effects of porous asphalt surfacing and barriers on traffic noise, Applied Acoustics 58 (1999) 351-377.

[7] O. Maeso, J. J. Aznárez, Estrategias para la reducción del impacto acústico en el entorno de carreteras. Una aplicación del Método de los Elementos de Contorno, Universidad de Las Palmas de Gran Canaria, 2005. URL: http://hdl.handle.net/10553/1500, ISBN: 84-689-0340-X.

[8] D. Greiner, B. Galván, J. Periaux, N. Gauger, K. Giannakoglou, G. Winter, Advances in Evolutionary and Deterministic Methods for Design, Optimization and Control in Engineering and Sciences; Computational Methods in Applied Sciences, volume 36, Springer, 2015. 
[9] D. Greiner, J. J. Aznárez, O. Maeso, G. Winter, Shape design of noise barriers using Evolutionary optimization and Boundary Element, in: B.H.V. Topping, G. Montero, R. Montenegro (Ed.), Proceedings of the Fifth International Conference on Engineering Computational Technology, Civil-Comp Press, Stirlingshire, UK, Paper 43, 2006.

[10] D. Greiner, B. Galván, J. J. Aznárez, O. Maeso, G. Winter, Robust design of noise attenuation barriers with Evolutionary multiobjective algorithms and the Boundary Element Method, in: M. Ehrgott et al. (Ed.), Lectures Notes in Computer Science, volume 5467, 2009, pp. 261-274.

[11] D. Greiner, J. J. Aznárez, O. Maeso, G. Winter, Single- and multi-objective shape design of Ynoise barriers using Evolutinary computation and Boundary Elements, Advances in Engineering Software 41 (2010) 368-378.

[12] K. Deb, S. Bandaru, D. Greiner, A. Gaspar-Cunha, C. C. Tutum, An integrated approach to automated innovization for discovering useful design principles: Case studies from engineering, Applied Soft Computing 15 (2014) 42-56.

[13] R. Toledo, J. J. Aznárez, O. Maeso, D. Greiner, Optimization of thin noise barrier designs using Evolutionary Algorithms and a Dual BEM Formulation, Journal of Sound and Vibration 334 (2015) 219-238.

[14] D. Duhamel, Shape optimization of noise barriers using genetic algorithms, Journal of Sound and Vibration 297 (2006) 432-443.

[15] M. Baulac, J. Defrance, P. Jean, Optimization of multiple edge barriers with genetic algorithms coupled with a Nelder-Mead local search, Journal of Sound and Vibration 300 (2007) 71-87.

[16] M. Baulac, J. Defrance, P. Jean, Optimisation with genetic algorithm of the acoustic performance of T-shaped noise barriers with a reactive top surface, Applied Acoustics 69 (2008) 332-342.

[17] S. Grubeša, K. Jambrošić, H. Domitrović, Noise barriers with varying cross-section optimized by genetic algorithms, Applied Acoustics 73 (2012) 1129-1137.

[18] L. A. de Lacerda, L. C. Wrobel, W. J. Mansur, A dual boundary element formulation for sound porpagation around barriers over an impedance plane, Journal of Sound and Vibration 202(2) (1997) 235-247.

[19] M. R. Schroeder, Diffuse sound reflection by maximum-length sequences, Journal of the Acoustical Society of America 57(1) (1975) 149-150.

[20] M. R. Schroeder, Binaural dissimilarity and optimum ceilings for concert halls: More lateral sound diffusion, Journal of the Acoustical Society of America 65(4) (1979) 958-963.

[21] T. J. Cox, P. D'Antonio, Acoustic Absorbers and Diffusers, $2^{\text {nd }}$ ed., Abington, Oxfordshire: Taylor \& Francis, 2009.

[22] M. R. Monazzam, Y. W. Lam, Performance of profiled single noise barriers covered with quadratic residue diffusers, Applied Acoustics 66 (2005) 709-730. 
[23] M. R. Monazzam, Y. W. Lam, Performance of T-shape barriers with top surface covered with absorptive quadratic residue diffusers, Applied Acoustics 69 (2008) 93-109.

[24] M. R. Monazzam, P. Nassiri, Contribution of quadratic residue diffusers to efficiency of tilted profile parallel highway noise barriers, Iranian Journal of Environmental Health Science \& Engineering 6 (2009) 271-284.

[25] M. Naderzadeh, M. R. Monazzam, P. Nassiri, S. M. B. Fard, Application of perforated sheets to improve the efficiency of reactive profiled noise barriers, Applied Acoustics 72 (2011) 393-398.

[26] M. R. Monazzam, M. Naderzadeh, P. Nassiri, S. M. B. Fard, Performance of environmental T-shape noise barriers covered with primitive root diffusers, Archives of Acoustics 35 (2010) $565-578$.

[27] T. Okubo, K. Fujiwara, Efficiency of a noise barrier on the ground with an acoustical soft cylindrical edge, Journal of Sound and Vibration 216 (1998) 771-790.

[28] T. Okubo, K. Fujiwara, Efficiency of a noise barrier with an acoustically soft cylindrical edge for practical use, Journal of the Acoustical Society of America 105 (1999) 3326-3335.

[29] A. Burton, G. Miller, The application of integral equation methods to the numerical solution of some exterior boundary-value problems, Proceedings of the Royal Society of London 323 (1971) 201-210.

[30] T. Ishizuka, K. Fujiwara, Performance of noise barriers with varios edge shapes and acoustical conditions, Applied Acoustics 65 (2004) 125-141.

[31] T. Okubo, Edge-modified noise barriers, J INCE Jpn 28 (2004) 317-322.

[32] R. Kress, Minimizing the condition number of boundary integral operators in acoustic and electromagnetic scattering, The Quarterly Journal of Mechanics \& Applied Mathematics 38 (1985) 323-341.

[33] S. Amini, On the choice of the coupling parameter in boundary integral formulations of the exterior acoustic problem, Applicable Analysis. An International Journal 35 (1990) 75-92.

[34] A. Tadeu, J. António, P. Amado Mendes, L. Godinho, Sound pressure level attenuation provided by thin rigid screens coupled to tall buildings, Journal of Sound and Vibration 304 (2007) 479-496.

[35] J. D. R. Bordón, J. J. Aznárez, O. Maeso, A 2D BEM-FEM approach for time harmonic fluidstructure interaction analysis of thin elastic bodies, Engineering Analysis with Boundary Elements 43 (2014) 19-29.

[36] J. F. Luo, Y. J. Liu, J. Berger, Analysis of two-dimensional thin structures (from micro- to nano-scales) using the boundary element method, Computational Mechanics 22 (1998) 404-412.

[37] J. Domínguez, Boundary elements in dynamics, CMP Elsevier, 1993. 
[38] A. Portela, M. H. Aliabadi, D. P. Rooke, The dual boundary element method: Effective implementation for crack problems, International Journal for Numerical Methods in Engineering 33 (1992) 1269-1287.

[39] A. Sáez, R. Gallego, J. Domínguez, Hypersingular quarter-point boundary elements for crack problems, International Journal for Numerical Methods in Engineering 38 (1995) 1681-1701.

[40] M. Wall, GAlib: A C++ Library of Genetic Algorithm Components (http://lancet.mit.edu/ga/), Mechanical Engineering Department, Massachusetts Institute of Technology, 1996.

[41] UNE-EN 1793, Road traffic noise reduction devices. Test method for determining the acoustic performance. Part 3: Normalized traffic noise spectrum, 1997.

[42] D. Whitley, J. Kauth, GENITOR: A different genetic algorithm, Technical Report CS-88-101, Colorado State University. Department of Computer Science, Fort Collins, 1988.

[43] G. Syswerda, A Study of Reproduction in Generational and Steady-State Genetic Algorithms, in: Proceedings of the Foundations of Genetic Algorithms (FOGA), (Ed.) Gregory J. E. Rawling, Morgan Kaufmann Publishers, San Mateo, California, 1991, pp. 94-101. 